\title{
Sigmificado psicológico de los constructos novios, amigovios y free para jóvenes Pachuqueños
}

Psychological meaning of the constructs dating, fuckfriends and free in Pachucas's young people

\author{
Aleyda Vizzuetth Herrera \\ Melissa García Meraz \\ Rebeca María Elena Guzmán Saldaña \\ Universidad Autónoma del Estado de Hidalgo
}

\section{Resumen}

Con el objetivo de conocer el significado psicológico de los constructos novios, amigovios y free, en la presente investigación se trabajó con base en el uso de la técnica de redes semánticas naturales (Figueroa, González, \& Solís, 1981); pero se utilizó el procedimiento y nomenclatura propuestos por Reyes-Lagunes (1993). Los estímulos se aplicaron a un total de 230 participantes, de los cuales 32 han mantenido una relación de amigovios, 29 han tenido una relación free, 74 tienen un noviazgo y 95 personas manifestaron no tener ninguna relación. El instrumento fue aplicado de manera individual y colectiva en diferentes escuelas del municipio de Pachuca, Hidalgo, garantizando el anonimato. Los resultados reflejan que el estímulo amigovios está definido por palabras como amistad, cariño, diversión, comprensión y confianza. El estímulo free está definido por aspectos como libertad, sexo, amistad, diversión y "no". Mientras que el estímulo novios está definido por palabras como amor, cariño, honestidad, respeto, comprensión y amistad. Según Miller, Vandome y McBrewster (2010), las relaciones casuales son diferentes del sexo casual. En las relaciones que son descritas como sexo casual, no existe un interés emocional por la persona, no son amigos ni se tienen cariño o amor. El sexo casual es de una sola noche (one night stand). En cambio, los amigovios sí generan y esperan de su relación cariño y amor; se carece de la parte del compromiso, pero no de la intimidad: amor y cariño conviven con la informalidad. Por ello, los resultados son discutidos en términos de los cambios culturales y sociales que han atravesado el inicio y la disolución de las relaciones de pareja.

\footnotetext{
Palabras Clave: amigovios, free, novios, significado psicológico, pareja.
}

Nota de Autor:

Aleyda Vizzuetth Herrera, Departamento de Psicología, Universidad Autónoma del Estado de Hidalgo; Melissa García Meraz, Departamento de Psicología, Universidad Autónoma del Estado de Hidalgo; Rebeca María Elena Guzmán Saldaña, Departamento de Psicología, Universidad Autónoma del Estado de Hidalgo.

La correspondencia en relación con este artículo debe dirigirse a Melissa García Meraz, Departamento de Psicología, Universidad Autónoma del Estado de Hidalgo, Ex Hacienda La Concepción s/n Tilcuautla, Hidalgo, C.P. 42160, Municipio de San Agustín Tlaxcala.

Correo electrónico: melissaunam @yahoo.com.mx 


\begin{abstract}
In order to identify the psychological meaning of the constructs: boy/girlfriend, fuckfriend, and free a questionnaire was administrated, following the semantic networks technique (Figueroa, Gonzáles, \& Solís, 1981), but the procedure selected was proposed by Reyes (1993). The questionnaire was applied to a 230 participants, in which 32 of the participants had a relationship called as fuck friend, 29 had a relationship called free, 74 were in a courtship and 95 had no relationship. The instruments were applied individually and collectively in different schools in the municipality of Pachuca, Hidalgo; in all the cases anonymity was ensured. The results show that the stimulus fuck friend is defined by words like friendship, love, fun, understanding, and trust. The concept of a free is defined by aspects such as freedom, sex, friendship, fun and "no". While boyfriends stimulus is defined by words like love, affection, honesty, respect, understanding and friendship. As mentioned Miller, Vandome and McBrewster (2010), casual relationships and casual sex are different. In relationships that are described as casual sex, there is no emotional interest by the person, they are not friends, and they do not give affection or love. Casual sex is a one night stand. In contrast, people that are involved in fuck friend relationships expect affection and love, the relationship lacks the commitment but not the part of intimacy: love and affection are in an informal relationship. Therefore, the results are discussed in terms of cultural and social changes that are present in the beginning and dissolution of relationships.
\end{abstract}

Keywords: fuck friend, free, courtship, psychological meaning, couple.

De acuerdo con Bauman (2006), las relaciones amorosas no pueden ser definidas como tal en la actualidad, por ello, propone que comencemos a llamarlas "conexiones". Estas relaciones están caracterizadas por un rompimiento con lo antiguo; se definen por ser diferentes a las relaciones del pasado, sin compromiso a largo plazo y marcadas por la ligereza del momento, del sentido pragmático de ser hechas a la medida y para el corto plazo. Relaciones verdaderamente útiles sólo para el momento, de fácil entrada, pero también de fácil salida, que fluctúan entre los encuentros ocasionales y las relaciones a muy corto plazo. Casi como una ligereza de emociones, se describen como conexiones que se realizan en el mundo moderno y que carecen del compromiso que antaño definiera a las relaciones de pareja. ¿Qué significa esto?, ¿acaso los individuos estamos perdiendo nuestra capacidad de relacionarnos con otros a largo plazo, de comprometernos?
A diferencia del pasado, las relaciones de pareja tienen un inicio, un desarrollo y un desenlace distinto. Ya no podemos caracterizarlas por representar un compromiso, marcado por la llegada del matrimonio y que sólo finaliza con la muerte de uno de los cónyuges, donde los comportamientos sexuales sólo tienen cabida dentro del lecho marital. Más allá de esto, las relaciones han redifinido, han mostrado lo polifacético de la sexualidad y del ser humano mismo. En la actualidad, las relaciones entre los jóvenes son definidas como relaciones de noviazgo, relaciones de amigovios —las cuales mezclan tanto a los amigos como a los novios-, y las relaciones de tipo free - caracterizadas por la ausencia de amistad y de compromiso-. Sin embargo, poca investigación se ha realizado referente a conocer el significado que realmente tienen estos constructos para los jóvenes. ¿Significan una ausencia de compromiso y, con ello, la destrucción de todo tipo de relación por la simple instauración de cone- 
xiones o, en cambio, representan el establecimiento formal de relaciones a corto plazo?

Por ello y con base en el objetivo de conocer más acerca del significado psicológico de los constructos de novios, amigovios y free, se trabajó con la técnica de redes semánticas naturales (Figueroa et al.,1981); pero utilizando la propuesta de Reyes-Lagunes (1993) con un grupo de jóvenes de la ciudad de Pachuca.

\section{Relaciones formales versus relaciones in- formales}

En la actualidad existe un renovado interés por conocer aquellos comportamientos que se generan dentro de las relaciones románticas y cómo éstas pueden ser catalogadas. En sí, dichas relaciones pueden clasificarse como formales e informales. Entre las primeras se encuentran las relaciones de noviazgo y matrimonio. En las segundas, relaciones sin compromiso, como los amigovios y las relaciones de tipo ocasional, como el sexo casual.

El concepto amigovio ha sido aceptado desde hace más de 10 años, principalmente en Argentina. Sin embargo, el término existe en países como Colombia, Brasil, Uruguay y México. Usado de forma similar a conceptos como "vacilaciones", "vacilones", "machuques", "amigantes", "quirris", "entuques" o "amigos con derechos", implica la unión de amigos y novios. Los términos utilizados en América Latina son de difícil traducción. De hecho, la traducción a lengua alemana en función de seguir la idea de amigovio sería más bien una expresión: "wir sind Freunde und zugleich ein Paar", lo que sería "ser amigo y pareja" (Castellanos \& Accorsi, 2002; Mendoza \& Fraao, 2005; Paulinas, 2007). El término amigovio está compuesto por dos palabras: amigo y novio. Los amigovios no exigen tanto compromiso como el noviazgo, la constancia en la relación es esporádica, ya que sólo implica pasarla bien con alguien (Paulinas, 2007).
Los amigovios no necesariamente se casan, pero se mantienen cercanos; la relación no se mantiene en secreto. En la actualidad, los amigovios muestran un nuevo patrón de amistades sexuales que se viven en el momento, se comienza a considerar una alternativa más a las relaciones de pareja y no sólo un preámbulo del noviazgo (Paulinas, 2007). Los amigovios van más allá de los patrones heterosexuales de amistad y son definidos como relaciones genuinas, sinceras, que van más allá de los convencionalismos de la amistad y del noviazgo; la idea de los amigovios es como el decir "están bien, pero frente a la sociedad no son nada" (Camacho, 2004; Paulinas, 2007).

Las relaciones free están altamente asociadas al sexo casual y al one nigth stand por tal, los involucrados se pueden considerar como acompañantes sexuales de fin de semana (Nateras, 2004). En las relaciones que son descritas como sexo casual no existe un interés emocional por la persona, no son amigos ni se tienen cariño o amor. El sexo casual es de una sola noche (one night stand). En cambio, los amigovios sí conciben y esperan de su relación cariño y amor, la relación carece del compromiso, pero no de la intimidad: amor y cariño conviven con la informalidad.

Existe, sin embargo, un amplio rango de interacciones. Miller, Vandome y McBrewster (2010) y Camacho (2004), afirman que debe diferenciarse entre sexo casual y relaciones casuales. El sexo casual también es identificado como one night stand, implica una interacción que es simplemente física, con poco o nulo interés emocional, que se da como un encuentro sexual de una noche. En cambio, las relaciones casuales implican más de un encuentro. En estudios realizados por Wentland (2007), los participantes manifestaron que las mujeres envueltas en relaciones de one night stand sienten mayor libertad sexual al no tener que preocuparse por el compromiso o el potencial de la relación, sino más bien, enfocarse en su propio placer sexual. 
En inglés existe un mayor espectro terminológico para designar a este tipo de relaciones. Así, one night stand implica que se tiene una interacción de tipo sexual sin interés emocional y de sólo una noche. Un booty call se refiere a una situación donde una persona llama a otra simplemente con la intención de tener relaciones sexuales en ese momento. Mientras que un fuck friend alude a una persona con la cual se tienen relaciones sexuales y una amistad, pero no es una pareja formal. En español, este tipo de interacción es conocida como "amigos con beneficios" o como "amigovios".

En el estudio de Miller, Vandome y McBrewster (2010), 25\% de las mujeres entrevistadas reportó que tenía sexo casual, $7 \%$ un booty call y $19 \%$ un fuck friend. Sin embargo, las relaciones casuales e informales tienen su parte negativa. Algunos estudios han mostrado que dos terceras partes de la violencia ocurrida durante las citas (date rape), se da en las relaciones informales o casuales, mientras que sólo un tercio toma lugar en relaciones a largo plazo o formales (Kandel, 2003). Además, dichas relaciones conllevan desilusión, falta de claridad de la asertividad sexual, la irresponsabilidad y la infidelidad.

\section{Método}

\section{Objetivo}

Identificar el significado psicológico de las relaciones formales (noviazgo) e informales (free y amigovios) en un grupo de participantes de la ciudad de Pachuca, que reportó haber estado en una relación de noviazgo, una relación free, una relación de amigovios y sin relación alguna.

\section{Instrumentos}

El significado es el resultado de la experiencia y conocimientos que acumula un individuo. Desde un punto de vista más conductual, el significado se encuentra cuando se obtiene la misma reacción frente a un objeto (Osgood, Suci, \& Tannembaum, 1971). Dado que el significado es el productor de las conductas, se convierte en sí en un significado psicológico, determinado por un contexto específico. A la vez que esta conducta es la respuesta del conjunto de elementos cognitivos y afectivos que reflejan la cultura subjetiva que tiene el individuo (Szalay \& Bryson, 1973). Por ello se utilizó la técnica de redes semánticas naturales (Figueroa, González, \& Solís, 1981), usando la nomenclatura propuesta por Reyes-Lagunes (1993) para referirse a los valores obtenidos por la técnica de redes semánticas naturales original, con el fin de conocer el significado psicológico de las palabras "novios, amigovios y free".

Los participantes respondieron a 3 estímulos presentados de forma aleatoria: novios, amigovios y free. Además, incluía una pequeña sección donde los participantes anotaban su escolaridad, edad, sexo y qué tipo de relación tenían en ese momento.

Tabla 1

Distribución muestral

\begin{tabular}{cccc}
\hline \multicolumn{2}{c}{ Relaciones informales } & Relación formal & \multicolumn{2}{c}{ Sin relación } \\
\hline Amigovios & Free & Novios & Sin ningún tipo de relación \\
32 & 29 & 74 & 95 \\
\hline
\end{tabular}




\section{Participantes}

Se trabajó con 230 participantes, de los cuales 61 mantuvieron una relación informal, 74 mantenían un noviazgo y 95 individuos no tenían ninguna relación. Sin embargo, es necesario anotar que la muestra de relaciones informales se dividió en dos grupos, 32 recono- cieron su relación como amigovios y 29 como free. Los participantes se ubicaban en un rango de edad entre los 15 y 26 años, con una media de 18.2, su nivel escolar era de preparatoria y universidad; de ellos 100 eran hombres y 130 mujeres.

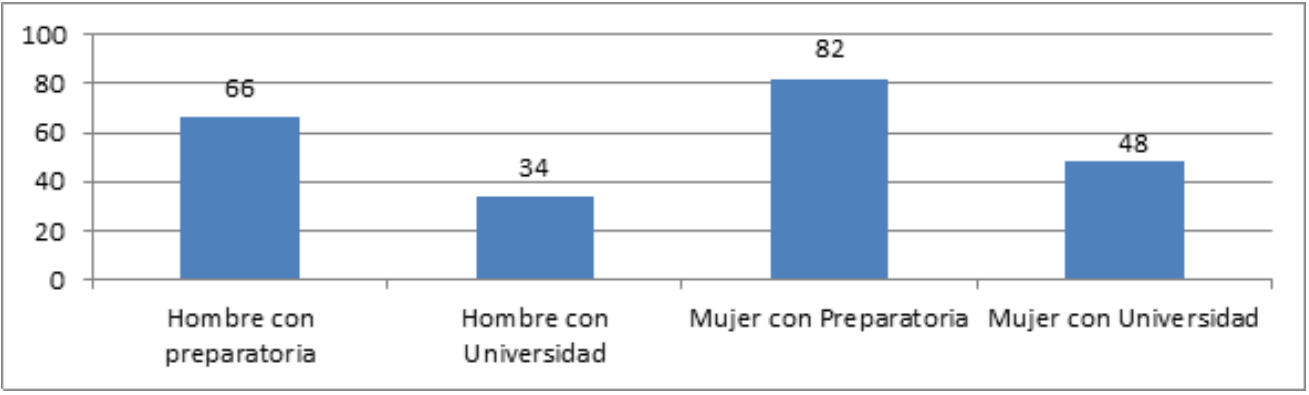

Figura 1. Número de participantes por escolaridad y sexo.

\section{Procedimiento}

El instrumento fue aplicado de manera individual y colectiva en diferentes escuelas del municipio de Pachuca, Hidalgo. Se solicitó a los participantes que contestaran las redes semánticas, colocando las primeras 5 palabras que relacionaran con los estímulos para, posteriormente, ordenarlas en forma de importancia: el valor 1 se asignaba a la palabra que ellos consideraran más relacionada con el estímulo y 5 a la menos relevante. La prueba tuvo una duración aproximada de 20 a 30 minutos y se garantizó el anonimato. Los resultados fueron capturados en el programa Excel y analizados por medio de la técnica de Reyes-Lagunes (1993).

\section{Resultados}

La tabla 2 muestra los diferentes pesos semánticos obtenidos por cada uno de los grupos. Aquí podemos observar que el mayor peso semántico en los cuatro grupos estudiados lo obtiene la definidora "amistad". Por otro lado, solo el grupo soltero difiere en la posición de "diversión" y "cariño", sin embargo, éstas siguen siendo las definidoras en segundo y tercer lugar, respectivamente. Otras definidoras que todos los grupos comparten, aunque con diferente orden jerárquico, son palabras como "libertad", "no compromiso" y "besos".

En la tabla 3 podemos observar las diferencias percibidas en cada uno de los grupos evaluados. Es interesante que a diferencia del estímulo amigovios en el estímulo free se encuentran mayores diferencias en el orden jerárquico; si bien las principales en todos los grupos fueron "libertad", "diversión", "sexo" y "no compromiso", también fueron las únicas definidoras comunes en los cuatro grupos.

Finalmente, en la tabla 4 se observan los pesos semánticos obtenidos por los 4 grupos para el estímulo novios. Los participantes parecen estar de acuerdo en que la principal definidora para el estímulo es "amor"; no obstante, a 
Tabla 2

Pesos semánticos para el estímulo amigovios dado por los 4 grupos

\begin{tabular}{llllllll}
\hline \multicolumn{1}{c}{ Amigovios } & & & \multicolumn{2}{c}{ Amigovios } & & & \\
Free & & & Novios & & \multicolumn{2}{c}{ Solteros } \\
\hline Definidora & PS & Definidora & PS & Definidora & PS & Definidora & PS \\
Amistad & 85 & Amistad & 49 & Amistad & 112 & Amistad & 134 \\
Cariño & 32 & Cariño & 27 & Cariño & 74 & Diversión & 102 \\
Diversión & 31 & Diversión & 27 & Diversión & 71 & Libertad & 80 \\
Confianza & 19 & Comprensión & 19 & Libertad & 62 & Cariño & 79 \\
Compañía & 17 & Besos & 16 & Besos & 60 & No compromiso & 39 \\
Libertad & 15 & No compromiso & 15 & No compromiso & 51 & Besos & 36 \\
Amor & 14 & Libertad & 13 & Nada & 36 & Confianza & 33 \\
No compromiso & 13 & Sexo & 13 & Atracción & 33 & Juego & 27 \\
Besos & 12 & Respeto & 10 & Sexo & 27 & Nada & 20 \\
Novios & 12 & Pasatiempo & 9 & Compañía & 23 & Respeto & 19 \\
Sexo & 11 & Novios & 8 & Comprensión & 23 & Amor & 15 \\
Respeto & 10 & Sinceridad & 8 & Informalidad & 23 & Derechos \\
\hline
\end{tabular}

\section{Tabla 3}

Pesos semánticos para el estímulo free dado por los 4 grupos

\begin{tabular}{llllllll}
\hline \multicolumn{1}{c}{ Amigovios } & & & \multicolumn{2}{c}{ Free } & & & \\
Free & & & \multicolumn{2}{c}{ Novios } & & \multicolumn{2}{c}{ Solteros } \\
\hline Definidora & PS & Definidora & PS & Definidora & PS & Definidora & PS \\
Diversión & 50 & Libertad & 57 & Libertad & 126 & Libertad & 194 \\
Libertad & 29 & Sexo & 33 & Sexo & 76 & Diversión & 96 \\
Amistad & 19 & Diversión & 30 & Diversión & 70 & No compromiso & 86 \\
No compromiso & 19 & Amistad & 28 & No compromiso & 64 & Amistad & 35 \\
Beso & 18 & No compromiso & 22 & Besos & 57 & Besos & 25 \\
Sexo & 16 & Pasatiempo & 15 & Juego & 34 & Inmadurez & 25 \\
Sin presión & 15 & Faje & 11 & Nada & 34 & Momento & 24 \\
Atracción & 9 & Gratis & 11 & Atracción & 32 & Cariño & 21 \\
Juego & 9 & Aventura & 9 & Fácil & 29 & Infidelidad & 21 \\
Pasión & 9 & Placer & 9 & Sin amor & 26 & Pasar el rato & 21 \\
\hline
\end{tabular}

pesar de ser nombrada por todos los grupos, cada uno consideró, en diferente orden jerárquico, definidoras como "cariño", "compromiso", "confianza" y "respeto". Mientras que sobresale la ausencia de la palabra sexo. 
Tabla 4

Pesos semánticos para el estímulo novios dado por los 4 grupos

\begin{tabular}{llllllll}
\hline \multicolumn{1}{c}{ Amigovios } & & & \multicolumn{2}{c}{ Novios } & & & \\
Free & & & \multicolumn{2}{c}{ Solteros } \\
\hline Definidora & PS & Definidora & PS & Definidora & PS & Definidora & PS \\
Amor & 77 & Amor & 91 & Amor & 256 & Amor & 258 \\
Cariño & 29 & Confianza & 30 & Respeto & 71 & Respeto & 102 \\
Honestidad & 20 & Respeto & 20 & Cariño & 67 & Cariño & 73 \\
Compañía & 18 & Cariño & 19 & Confiable & 65 & Confianza & 63 \\
Compromiso & 18 & Besos & 18 & Comprensión & 46 & Compromiso & 59 \\
Confianza & 17 & Compañía & 18 & Fidelidad & 40 & Relación & 42 \\
Respeto & 16 & Amistad & 16 & Pareja & 33 & Fidelidad & 38 \\
Besos & 13 & Compromiso & 11 & Amistad & 32 & Compañía & 37 \\
Relación & 13 & Chicos & 10 & Besos & 32 & Amistad & 36 \\
Felicidad & 12 & Felicidad & 9 & Compromiso & 31 & Felicidad & 32 \\
Fidelidad & 11 & Abrazos & 8 & Honestidad & 24 & Apoyo & 28 \\
Lealtad & 10 & Comprensión & 8 & Relación & 24 & Comprensión & 28 \\
\hline
\end{tabular}

\section{Discusión}

En la actualidad, las relaciones casuales parecen estar de moda. Con una frecuencia cada vez mayor, las personas deciden entrar en relaciones informales. Los amigovios, free, LAT (relaciones de tipo Living Apart Together, es decir, estar juntos como pareja, pero mantener una residencia separada) y demás configuraciones de la relación de pareja, muestran una hipótesis que ya era predecible: los individuos han modificado su idea tradicional acerca de la vida en pareja, del matrimonio y el divorcio e, incluso, de la idea misma de estar juntos.

De manera constante y a una edad temprana, los sujetos evalúan de forma negativa el matrimonio y, con ello, de modo más positivo el permanecer solteros, casarse a una edad adulta, favorecen la unión libre y el sexo premarital (García \& Reyes-Lagunes, 2009). Sin embargo, aun y cuando las personas tengan una opinión menos positiva acerca del matrimonio, lo cierto es que siguen intentando una y otra vez encon- trar una pareja, ya sea en una relación formal como en el matrimonio o en relaciones de tipo más informal. La palabra o término amigovio implica una relación entre dos amigos y conlleva ciertos beneficios relacionados a conductas eróticas o sexuales. Cuando se tiene una relación de amigovio se puede o no tener relaciones sexuales, aunque sí hay besos, caricias, arrumacos y lo que comúnmente denominaríamos "fajes". Este sentido de "amigos con derecho" o "amigos con beneficios" implica que se tiene derecho a ciertas conductas sexuales que usualmente no se tiene con los amigos.

Quizás de ahí el hecho de que al buscar el significado psicológico de la palabra amigovios (véase figura 2), nos encontremos la palabra sexo, pero también todos aquellos comportamientos que implican besos y caricias. La palabra amigovios vincula lo más hermoso de los amigos: amistad, cariño y diversión, así como características de los novios: besos, caricias y cariño. ¿Qué es lo que este término deja fuera?: la formalidad y el compromiso. Esto es evidente, 


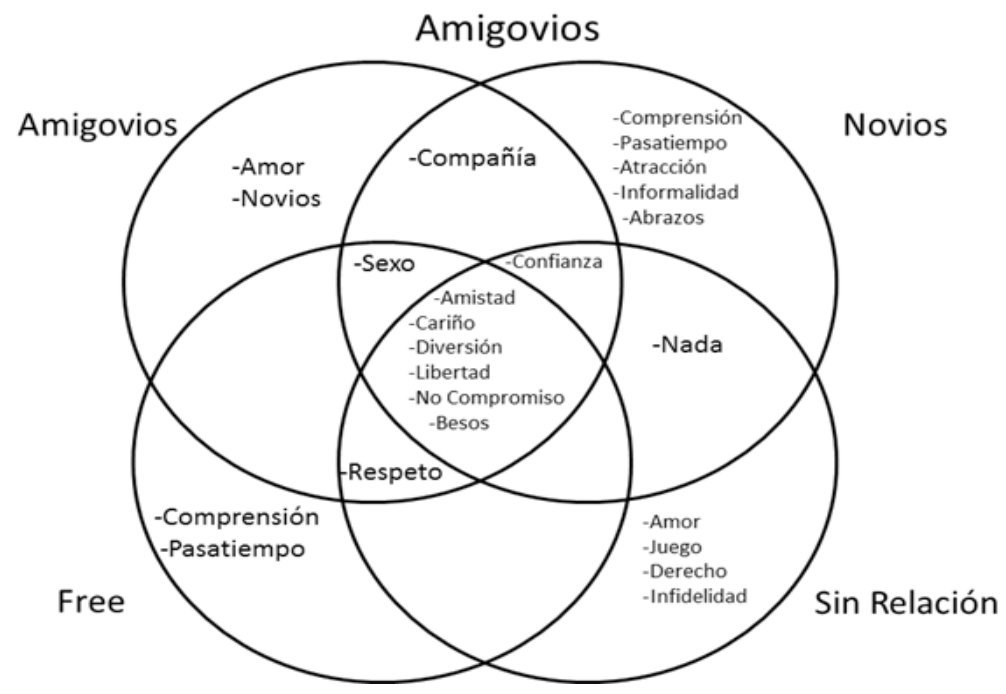

Figura 2. Significado psicológico de amigovios en los cuatro grupos.

sobre todo, en el caso de los novios; para ellos, la relación de amigovios, sí comprende besos y amistad y demás características positivas pero, sobre todo, informalidad y "nada", no se puede esperar de esta relación algo más. Es interesante anotar que, a diferencia del estímulo amigovios, en el estímulo free se encuentran mayores diferencias en el orden jerárquico de las palabras definidoras, si bien las principales en todos los grupos fueron "libertad", "diversión," "sexo" y "no compromiso", también fueron las únicas definidoras comunes en los cuatro grupos.

De una relación de amigovios se pueden esperar besos, caricias, libertad, sexo y comprensión. De hecho, sorprende observar que aparezca de manera consistente la palabra amor. Parece ser que la idea de sexualidad y besos con un amigo, no deja de lado la idea de amor. Después de todo se puede y se ama a un amigo.

Bajo la teorización de Sternberg (2000), las relaciones de pareja están basadas en tres componentes: intimidad, pasión y compromiso. Con esta idea, nos percatamos de la existencia de intimidad en las relaciones informales, donde también existe felicidad, reconocimiento de amor, entendimiento, cariño y demás en convivencia con la idea de pasión, sexualidad, besos y faje. Sin embargo, el compromiso no se encuentra. Para el autor, el compromiso es el elemento cognoscitivo que apoya la decisión de amar y permanecer con el ser amado. Es un compromiso de permanecer con una persona, de estar satisfecho en la relación y mantener el vínculo de amor a largo plazo. En el caso de los amigovios, lo anterior no puede ni debe darse. Recordemos que Sternberg (2000) establece diferentes vías que puede tomar una relación cuando los diferentes componentes (intimidad, pasión y compromiso) se mezclan. En el caso de los amigovios, hablamos de una relación tipo amor romántico, donde únicamente se presenta la intimidad y la pasión. Carece del sentido de compromiso, de hecho, palabras que implican esta falta de compromiso evidencian dicha tendencia: pasatiempo, juego, no compromiso, engaño, irresponsabilidad y, de manera especial, la idea de desilusión en los amigovios.

Quizás, la situación en este caso se deba a que los amigovios, en realidad, podrían en 
algunos momentos desear algo más, pero una relación que comienza bajo el principio de no compromiso por lo común no se modifica. En Estados Unidos, el término amigovio no tiene traducción, difícilmente podría decirse girlfriend. De hecho, el sentido de novio o novia en inglés ya implica la amistad. Por ello, el término más usado es "amigos con derecho" (friends with benefits). Otro término de uso popularizado entre la población no muy retomado en la literatura, es el término fuck friends. A diferencia del primero, este término es más claro acerca de la idea del sexo dentro de esta relación.

De hecho, al comparar los resultados acerca de los significados de amigovios versus free, se encontró que para todos los participantes de esta última categoría, la definición es el sexo, que además implica para los participantes sexo. Además, implica aventura y placer. Podría pensarse que dicho término está más relacionado con los fuck friend, pero lo cierto es que mientras éstos consideran tener relaciones de mayor tiempo; parece que la idea de free está más vinculada a una situación momentánea. Tal relación difiere con la de amigovios e implica un sentido más sexual, pasajero y momentáneo; un ligue o un encuentro casual. Tan es así que las palabras descritas por los participantes son aventura, placer, faje, sexo, momento, pasión, atracción y sin amor. En el caso de los novios esto es más constante: nada, fácil, sin amor e irresponsabilidad. Aquellos que no tienen una relación también pensarían que puede haber inmadurez e infidelidad en las relaciones free (véase figura 3).

Tomando en consideración a Sternberg (2000), la relación free se consideraría como un enamoramiento, donde el único componente es la pasión. Entendamos pues, que el enamoramiento no debe ser confundido con amor. Recordemos que el amor es el fin al que busca llegar una relación de pareja considerada plena, y para que esta relación sea plena son necesarios la intimidad, la pasión y el compromiso. Por ese motivo, el enamoramiento, al estar compuesto únicamente por la pasión, se considera más fugaz, así como en las relaciones free donde se busca principalmente la satisfacción sexual.

Las relaciones casuales tienen varias implicaciones. De acuerdo con Wentland (2007), cuando las mujeres se encuentran en relaciones casuales frecuentemente tienen menos comunicación sexual con el otro, también son

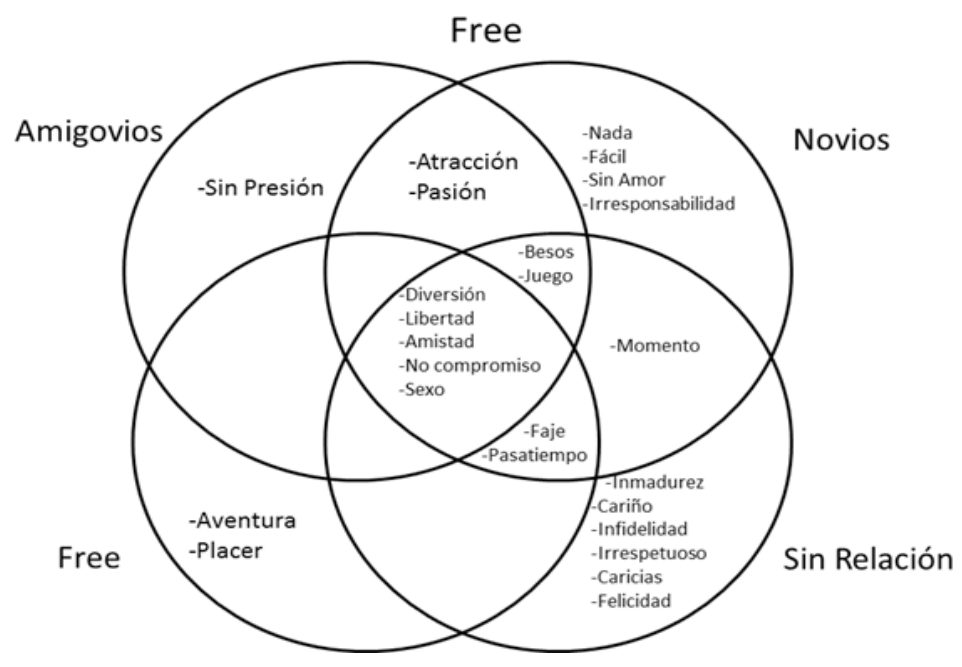

Figura 3. Significado psicológico de free en los cuatro grupos. 
más accesibles al sexo casual dentro de las citas. De acuerdo con el autor, quizás lo que suceda es que las mujeres temen verse demasiado asertivas con referencia al sexo, pues las mujeres en relaciones casuales son más propensas a buscar el sexo que las mujeres en relaciones formales. Parece que la informalidad les da la pauta para poder pronunciarse a favor del sexo. Si la cultura y las normas nos dicen que las mujeres deben guardar "recato" en las relaciones y no mostrar su interés por el sexo, aquellas en relaciones casuales sí muestran su interés por el sexo.

Los roles tradicionales de género dentro de las citas de noviazgo implican que las mujeres siguen un patrón tradicional según el cual ellas son las encargadas de soportar la relación, son cariñosas, afectivas, se ríen de los chistes del chico, etc., mientras que ellos son los encargados de la parte activa, ellos indican hacia donde irá la relación, pagan, invitan, recogen a la chica, le dan un regalo, etc. Con base en este patrón, los chicos proponen el inicio de las relaciones sexuales, buscan desarrollar estrategias encaminadas a tal fin. Mientras que las mujeres son educadas para evitar o negarse al sexo y, por ende, a no mostrar su interés en ello (García \& Romero, 2011).

En cambio, el noviazgo involucra situaciones diferentes. El significado psicológico de novios implica que las personas unen las palabras como amor, cariño, compromiso, confianza, respeto y besos. La relación de novios comprende los tres componentes de la teoría triangular del amor de Sternberg (2000): intimidad, pasión y compromiso. Quizás aquí el factor de compromiso es el más anunciado. En este caso son los novios los que añaden más características a esta situación, hay fidelidad, honestidad, relación, ternura, formalidad, responsabilidad y apoyo. De hecho, tanto novios como solteros incluyen el sexo, pero los que se encuentran en relaciones casuales no. Para ellos, la informalidad implica la sexualidad abiertamente, la formalidad no (véase figura 4).

En el contexto anterior, varios son los autores para quienes la violencia sexual es un hecho frecuente dentro de las citas de noviazgo, comúnmente conocido como date rape. Este tipo de abuso puede darse tanto en una relación seria como en una casual; el victimario

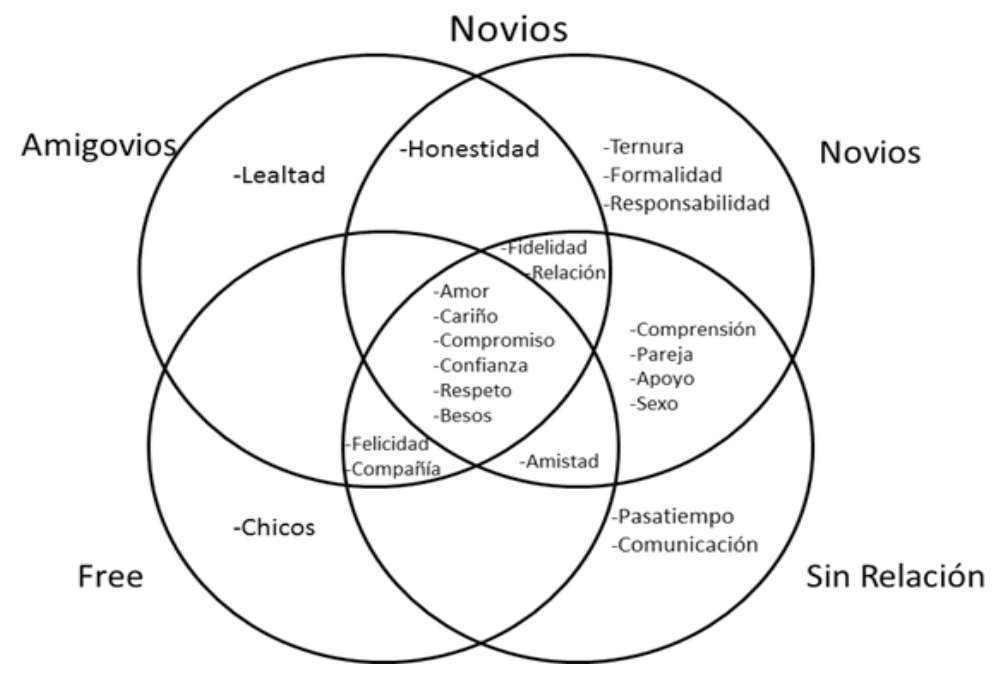

Figura 4. Significado psicológico de novios en los cuatro grupos. 
podría ser un amigo, un compañero, estudiante, compañero del trabajo, o incluso un compañero de viaje.

La desvinculación del sexo y del noviazgo es tal, que las mujeres no lo mencionan. Esto concuerda con lo reportado acerca de los roles de género tradicionales en hombres y mujeres; mientras que los hombres pueden manifestar de forma abierta su interés en el sexo, las mujeres no. Entre las Investigaciones realizadas en México (García \& Reyes-Lagunes, 2004) han mostrado que los individuos asignan características positivas a estímulos como pareja, matrimonio y rematrimonio: amor, respeto, confianza, compromiso, amistad y fidelidad, pero, en todos los casos, dejan fuera palabras relacionadas con la sexualidad.

En la época contemporánea, la gama de colores e intensidades que dibujan las relaciones de pareja conlleva algo más que falta de interés o compromiso. La vida moderna nos llena de posibilidades acerca de la interacción. La desvinculación entre matrimonio y sexualidad dan cuenta de tal situación en terrenos diferentes. Relaciones casuales, de una noche, de una cita, que abren la posibilidad de la sexualidad y el erotismo. Relaciones que, si bien, son más que conexiones son seguramente relaciones de fácil término. Relaciones que abren la posibilidad de encontrar una forma de experimentar la idea misma de pareja.
Bauman, Z. (2006). Amor Líquido. Acerca de la fragilidad de los vínculos humanos. México: Fondo de Cultura Económica.

Camacho, J. (2004). Fidelidad e infidelidad de pareja. Buenos Aires: Dunken.

Castellanos, G. \& Accorsi, S. (2002). Género y sexualidad en Colombia y en Brasil. Buenos Aires: La Manzana de la Discordia.

Figueroa, J., González, R., \& Solís, V. (1981). Una aproximación al problema del significado: las redes semánticas. Revista Latinoamericana de Psicología, 13(3), 447-458.

García, M. M. \& Romero, P. A. (2011). Coerción sexual en el noviazgo. En R. Díaz y S. Robles (Eds.). Salud y Sexualidad. México: UNAM, CONACYT. AMEPSO.

García, M. M. \& Reyes-Lagunes, L. I. (2004). Amor y desamor: del matrimonio al divorcio. La Psicología Social en México, X. México: AMEPSO.

García, M. M. \& Reyes-Lagunes, L. I. (2009). Actitudes hacia el matrimonio y el divorcio: Diferencia por sexo, residencia y estatus de pareja. Psicología Iberoamericana, 17(1), 48-56.

Kandel, E. (2003). Understanding violence ( $2^{-a}$ ed). New Jersey: Lawrence Erlbaum associates.

Mendoza, I. \& Fraao, V. M. (2005). Berlitz Vorsicht Spanisch! München: Berlitz Publiching.

Miller, F. P., Vandome, A. F., \& McBrewster, J, (2010). Casual relationships. US: Publishing House Ltd.

Nateras, A. (2004). Los trazos de lo emergente juvenil urbano. En S. Arciga B. (Ed.). Del pensamiento social a la participación. Estudios de psicología social en México. México: SOMEPSO, Universidad Autónoma de Tlaxcala, UNAM, UAM-Iztapalapa.

Osgood, Ch., Suci, G., \&Tannembaum, T. (1971). The measurement of meaning. Chicago: The University of Illinois Press.

Paulinas (2007). Entre el goce, el afecto y los temores jóvenes y sexualidad. Bogotá: Editor Paulinas.

Reyes-Lagunes, I. (1993). Las redes semánticas naturales, su conceptualización y su utilización en la construcción de instrumentos. Revista de Psicología Social y Personalidad, 9(1), 81-97.

Sternberg, J. R. (2000). La experiencia del amor: la evolución de las relaciones amorosas a lo largo del tiempo. México: Paidos.

Szalay, L. \& Bryson, A. (1973). Measurement of psichocultural. Journal of personality and social psychology, 26(2), 166-167.

Wentland, J. J. (2007). Sexual pleasure orientation in heterosexual women. University of Guelph thesis. University of Guelph. Dept. of Family Relations and Applied Nutrition, University of Guelph. School of Graduate Studies. 